\title{
Virtual Homage to the Dead: An Analysis of Digital Memorials in the Social Web
}

\author{
Aron Daniel Lopes, Cristiano Maciel, and Vinicius Carvalho Pereira
}

Universidade Federal de Mato Grosso (UFMT)

Laboratório de Ambientes Virtuais Interativos (LAVI)

Av. Fernando Corrêa da Costa, n²367 - Cuiabá/MT - Brazil -78060-000

aronlopes@gmail.com, cmaciel@ufmt.br, vinicius.carpe@yahoo.fr

\begin{abstract}
Considering that many real-world cultural practices are now migrating to virtual environments, the expression of mourning and bereavement is also being imported to the virtual world, by means of the so-called digital memorials, supported by increasingly new and complex technologies. In this paper, we undertake a literature review on real and digital memorials, as well as social networks. Then, through empirical observation of digital memorials in Brazil, we investigate if they have characteristics of the social web. Next, by means of an interaction test and a questionnaire, we analyze how users feel when interacting with digital memorials and their evaluation on the functionalities of those applications. Finally, we approach the difficulties found when studying this kind of tools and our perspectives for future works.
\end{abstract}

Keywords: digital memorials, social web, social networks, death.

\section{Introduction}

In modern societies, digital technologies are increasingly embedded in our daily activities, thus changing the way we see the world and act upon it. That leads realworld social practices to migrate into virtual environments and acquire new meanings, including cultural expressions triggered by someone's death.

Death is an unavoidable event which entails both sacred and profane rites, most often performed by bereaved people. Among those rites, we can find wakes, funerals, burials, requiem masses, prayers and the building of memorials.

However, as cultural practices migrate from the real world into the virtual one, such rites may change too. Virtual applications that model real memorials in virtual places (herein called virtual memorials, digital memorials or cyber memorials) are becoming more popular, especially in the social web. Such software programs are intended to allow users to pay digital homage to deceased people.

In Brazil, the development of this kind of technology is on the rise, which reinforces the urgent want for more research on this theme. Related discussions must address potential users' needs and wishes concerning those memorials, but it is also necessary to bear in mind that such technologies involve big emotional loads. 
Through literature review and empirical observation on digital memorials, this paper addresses issues related to the shift of memorials into the digital world, by investigating recent digital memorials in the Brazilian social web. Then, we analyze how those programs work and, by means of an interaction test followed by a questionnaire, we investigate how users feel when interacting with those applications.

After this introduction, we present the results of the literature review concerning digital memorials. Then, we describe the research methodology in detail and we present the results of the investigation on the software programs and the results from the experiments with users. Finally, there are the ending discussions and the references.

\section{Digital Memorials}

In the physical world, a memorial is "something, such as a monument or holiday, intended to celebrate or honor the memory of a person or an event" [2]. The practice of funeral rites, which involves building memorials in many societies, is considered by Structural Anthropology as the turning point between the state of nature and the state of culture [4]. Therefore, we are no longer common animals and become members of the human culture when we realize death is a different stage in the life cycle, which requires specific practices. These funeral rites have not only hygienic reasons (removing the corpse lest an infection spreads). Instead, they are mingled with many symbolisms, such as fear of spirits, profanation taboos and requests for mercy and protection.

In the virtual world there are many cultural practices about death as well, which involve both the creation of new funeral rites and the migration of those already performed in the physical world. In this context, many social web software applications are being used to express bereavement and mourning, even if they were not designed for this purpose. Two main strategies are performed by users in social networks such as Orkut and Facebook to express sorrow, surprise or missing of a deceased person: a) posting messages on the webpage created by the user before he or she died; or b) creating memorial webpages for the dead [10].

According to [17], although death-related processes are mainly determined by religious and cultural practices, technology may have an important role as well, even if invisibly. "From seeking information online about available burial options to creating multimedia presentations for use at a memorial service, technology can inform, comfort, confront, and connect the bereaved in the years following a death" [17]. In terms of contributions, the authors also make statements that can be translated into guidelines for the design of processes that explicitly consider mortality, death and bereavement in the conception of a system [17].

Besides permitting tributes to people who have passed away, digital memorials provide users with an environment where they can gather and share the deceased person's memories. Relatives may feel comfortable when they visit a social network profile of a lost kin and see there is lots of homage to that person [16] [11]. Furthermore, [29] states that contemporary digital memorials add "the benefits of rich 
media content and introducing a marked shift of authorship and intent into the process of cultivating and maintaining a postmortem identity".

When it comes to interaction provided by a memorial about someone's death, [23]'s taxonomy must be considered: the bereaved person's self-interaction, the interaction among living people about someone's death, and the interaction between the dead and the alive. Therefore, memorials and the materiality they give to bereavement meet not only the bereaved person's needs. They also meet the dead person's expectations that we should pay attention to him, honor him or simply realize what is happening to him [28].

After this introduction about the digital memorial theme and works related to the area, this research's methodology follows.

\section{Methodology}

This research began with a literature review on related works and on issues of death and cultural practices on the web, such as posthumous interaction[12], death-related taboos and beliefs [13], Facebook memorial pages [10] and virtual memorials in general [4].

Then, we researched on Brazilian social software applications that work as digital memorials and found the following recent platforms: iHeaven [9], SaudadeEterna [25] (Eternal Missing) and Memorial Digital [18] (Digital Memorial). According to [3], empirical observation on the web, the method we chose for this study, allows the researcher to identify users' expectations for future interactions in new technologies.

We began the observation by registering in the aforementioned social networks, but we were only successful to complete this process in iHeaven and SaudadeEterna. There was no feedback from Digital Memorial as to our registration.

Later on, we investigated iHeaven and SaudadeEterna to identify what characteristics of social web were present in those applications. By means of netethnography [22], we collected data that were quantitatively and qualitatively analyzed in the light of social software elements proposed by [26], [14] and [12].

Next, we applied an interaction test in Heaven with users who had never seen that application before. That test was intended to collect information on how users feel when interacting with the functionalities of such platform. 29 people from Cuiabá (a city in the Middle West of Brazil) aged between 18 and 30 years old participated in this stage of the research. The sample was composed of users from the Y or Internet Generation [27] in accordance with the sample of other researches on posthumous data by [13]. All the participants were undergraduate students in Computer Sciences, Psychology, Social Sciences and Social Communication.

The tests were taken individually and were composed of two steps: the first one consisted of a task-scenario with a set of tasks to be fulfilled by the users in the platform; the second one consisted of a questionnaire.Each guided interaction test took about 20 - 30 minutes and the tasks were divided in three main stages: identifying the tools, interacting with an existing memorial, and creating a memorial. 
In the stage of identification of the tools, the user navigated freely in a memorial, by looking at each functionality mentioned in the task-scenario (profile, updates, homage and photos). In the following stage, the user interacted with any memorial he chose by using the tools available on the platform, according to the instructions of the task-scenario. In the last stage, the user was asked to create a memorial in the platform for a friend, a relative or a public person, but only if they felt comfortable do so.

After the interaction test, each user was asked to answer a questionnaire on his socio-demographic profile, his evaluation on the functionalities of the application, how he felt when fulfilling the tasks described in the task-scenario, and how different are physical and digital memorials. The questionnaires were answered in 12.5 minutes in average.All participants in the interaction test and respondents to the questionnaire signed a term of consent, according to the Brazilian Legal Resolution 466/2012 [5]. The data from the questionnaire were analyzed quantitatively and qualitatively, beginning with the socio-demographic profile. Next, the other data were analyzed in two main dimensions: how the users felt when interacting with the memorials and their evaluation on the functionalities of the application.

As mentioned, in this research we investigated the digital memorials provided by the platforms iHeaven and SaudadeEterna, described as follows.

iHeaven: The software iHeaven models the concept of memorials through tombstones (using the Portuguese verb "jaz", which means "here lies" and alludes to the deceased person to whom the memorial was created). In iHeaven, a user can create many memorials, where he must fill in text fields with information on the deceased person, such as name, nationality, place/date of birth and death, and biography. In order to interact with a memorial profile, the user must first add this profile (similarly to adding friends in other social networks). This functionality also requires that the user says which kind of relationship he had with that person (friend, relative etc.). Many photos can be added to a memorial, even organized in galleries. It is also possible to "pray" for that person or leave him a candle or flowers, by clicking the correspondent button. The bereaved user has his own profile and can send messages to other users, add them as friends and see their last updates in the platform. In this platform memorials of public people are especially popular, such as singers and actors.

SaudadeEterna: The platform SaudadeEterna also allows for the creation of memorials for deceased people. In this application, after creating an account and logging in, the user create one or more memorials containing information about the dead person, such as name, date/place of birth and death, and biography, just like in iHeaven. In the digital memorial, it is also possible to add photos and videos. In this platform, the user can pay tributes to the dead person (posts on the memorial profile), send him gifts (hearts, angels and candles, for instance) and express condolences to the family of the dead. However, the user doesn't have a profile of his own: all the interaction takes place in the memorials. Besides, there is no messaging system for users to communicate among themselves.

In the following sections, the results from the research are presented. 


\section{Analysis of the Platform as to Social Web Elements}

In this section, we present the results of our analysis on iHeaven and SaudadeEterna regarding the presence of the following social web elements defined by [26] [14] [12]:

- Identity: in SaudadeEterna, users are not identified by profiles in specific webpages, and all the interaction takes place in the memorials. iHeaven has specific webpages for the tombstones and the users, which emphasizes identity in this social software.

- Relationships: in SaudadeEterna only memorial pages are modeled, thus there are neither relationships between users and memorials nor among users. iHeaven provides friendship relationships among users and, family/friend/idol relationships among users and memorials.

- Conversation: only iHeaven provides users with a messaging system.

- Groups: by means of memorials, both platforms allow users to form communities of interest (in this case, the deceased person).

- Recommendation: in iHeaven, the "score" of the memorial works as a recommendation sign for the users.

- Reputation: in both platforms, homage is paid to the memorials, thus rating them. In SaudadeEterna, reputation comes from the presents a memorial is given, such as hearts, angels, candles etc. The icons for those presents are displayed in the memorial page. In iHeaven, the same functionality is present, although reputation is measured in terms of candles, flowers or prayers to the dead.

The elements Presence, Sharing and Volition could not be found in any of those applications. However, more empirical research on similar platforms is necessary before one can say this is a characteristic of memorial social networks.Table 1 summarizes the analysis of iHeaven and SaudadeEterna as to Social Web Elements.

Table 1. Comparative table of social web elements

\begin{tabular}{l|l|l}
\hline Elements & SaudadeEterna & iHeaven \\
\hline Identity & Memorials & Memorials and users \\
Presence & Absent & Absent \\
Relationships & Absent & $\begin{array}{l}\text { Relationships between user and } \\
\text { memorial and among users }\end{array}$ \\
Conversation & Absent & Present \\
Groups & About memorials & About memorials \\
Reputation & Present & Present \\
Sharing & Absent & Absent \\
Recommendation & Absent & Present \\
Volition & Absent & Absent \\
\hline
\end{tabular}


This analysis proves that iHeaven contains more elements of social software. However, in this stage of the research, it proved difficult to identify elements of the social web in these software platforms. As the concepts of "social network for the alive" and "social network for the alive and the dead" are quite different, the categories of analysis of social web elements should be redesigned and broadened, so as to meet the specificities of those applications.

With regard to [23]'s categories of interaction about death, we can see that the two platforms herein analyzed provide interaction between the alive and the deceased and self-interaction for the bereaved. However, interaction among the alive about someone's death is only partially allowed by those applications.

\section{$5 \quad$ Interaction Test}

This section presents the results of the interaction test, based on the data from the test and from the subsequent questionnaire.

Each volunteer took the interaction test individually, assisted only by one of the researchers. During the test, some remarks were made by the users and these data are also herein analyzed.The subsequent questionnaire was composed of 23 questions (10 open and 13 closed). 18 of those questions are herein analyzed. We use the letter "U", followed by a number, to refer to a user.

In the following section, all the data collected are analyzed in three categories: users' profile, how the users felt when interacting with the platform and their analysis on the functionalities of the application.

\subsection{Users' Profile}

$82,75 \%$ of the users were men. Regarding religion, $44,82 \%$ said they did not have a religion, 37,93\% were Catholics, 3,44\% Buddhists, 6,89\% Protestants e and 6,89\% choice the option "other". Those who affirmed to have a religion were asked how often they performed rites of that religion. All participants said they sometimes did so.

$75,86 \%$ of the participants were students of Computer Sciences; 10,34\%, of Psychology; and 6,89\%, of Social Communication. The other 6,89\% took undergraduate courses in different areas. As previously mentioned, all users are part of Generation Y, aged between 18 and 30 years.

\subsection{How Users Felt After Interacting with iHeaven}

When asked how they felt after interacting with a memorial in iHeaven, 68,96\% of the users answered "tranquility"; 44,82\%, "uneasiness"; 31,03\%, "peace"; 27,58\%, "sadness"; 20,68\%, "happiness"; 20,68\%, "discomfort"; 3,44\%, "fear"; and 17,24\% "other". In this question, more than one alternative could be chosen.

U06 stated he felt in peace but sad, and added: "I felt as if I were visiting that person's grave, and paying homage to him with flowers and candles makes one think of that environment". U12, who interacted with the memorial with a public person he 
personally knew, answered that he felt happy and justified: "I felt very well, even because I found some well-known faces of people I miss. Meeting them again, even if in this social network, reminds me of the people I miss". Some users who chose the option "other" said they felt curious when accessing those memorials. U14 stated: "I would like to know the causa mortis. I know this is indiscreet, but I feel very curious!"

U20 chose the option "other" and added that he felt "respectful", also saying: "It is interesting to see people paying homage in such an unusual fashion, which must be respected". U29 chose the same option and added he felt "inspired to read about the deeds of an important person".

U5 added that he felt melancholic and "in the beginning, he [I] was uncomfortable, but later on he was tranquil, though a bit scared". Figure 1 depicts how the users felt after interacting with the memorials.

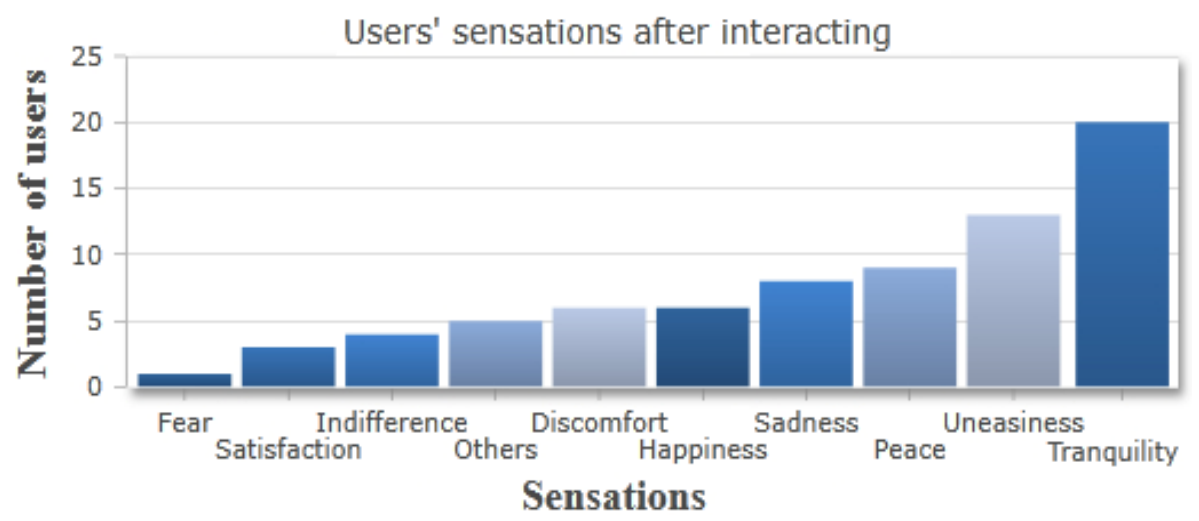

Fig. 1. Graph of how users felt after interacting with a digital memorial

\subsection{Users' Evaluation on the Functionalities of iHeaven}

In this subsection, we present the users' evaluation on some functionalities of iHeaven, especially focusing on the possibility of paying homage to the deceased person and the creation of a new memorial.

Paying Homage to the Deceased. With regard to homage paid in the memorials, such as messages, prayers, candles and flowers, 65,51\% of the users answered all those options had the same symbolic meaning. On the other hand, most of those $34,48 \%$ who said they had different values considered the option "message" as the most meaningful. U12 stated: "It was the most common option, I guess! I wrote what I really feel for the person I was paying homage to, instead of simply clicking the candle or prayer button.

U07 added that "messages show there are people who care so much about a dear person's death that they want to show their feelings in a public space". U16, during the interaction test, said the following before writing a message on a memorial: "I will 
not write that, it is disrespectful". In his speech, that user seems to consider a memorial as something sacred that must not be profaned [13].

There was also some dissension as to what functionalities are proper for a digital memorial. U04 said he "liked messages and prayers, but candles and flowers are needless". This opinion emphasizes the value of immaterial kinds of homage, instead of physical objects, although in iHeaventhere are only icons of those objects on the interface. Possibly, this opinion is related to the fact that messages and prayers can be understood as means of verbal communication someone dead might receive by supersensible means.

On the other hand, U06 affirmed that he "found the option praying superficial, because it was just clicking a button". E15 adds: "By clicking 'pray', you do not pray. You only pray by praying". This is very similar to the opinion of U11: "I found the option 'pray' irrelevant. It has no concrete meaning, it is just clicking a button and that is it, I believe there is no real expression in that". U19 verbally expressed his distaste for the option "pray": "Praying? This is wrong! Some people will be obsessed with that! They will think all they need to do is click that button". E19 said the virtual prayer does not replace praying in the real life.

Many users compared the option "pray" with the functionality "like" in Facebook. This was considered as a trivialization of praying, merely reduced to a click. U10 affirmed he "felt as if he were 'liking' the memorial. That was weird!"

The resistance to use the "prayer" functionality, if compared to the other homage options, is clearly related to the taboo of dehumanizing a religious action, which should demand concentration, time and cognitive effort, instead of easiness, speed and practicality, characteristics of social networks and mechanical procedures.

On the other hand, U09 had a different opinion on the "prayer" functionality: "I think all these things are intended to religious people. All a user is able to do in this social network excludes non-religious people. Memorials are made by all sorts of people, for all sorts of people". U07 added: "As I am an Atheist, it [the prayer option] does not make any sense to me".

Those answers show that even non-religious users do not feel comfortable when the platform addresses religious issues. It is necessary to rethink those functionalities and possibly create new ones to support people in the platform, irrespective of religion, as stated by U05: "All those tools are valid, but they represent only one religion: Christianity. Maybe new kinds of homage are necessary, or the existing ones should be turned into something more neutral".

This matter is twice hard to deal with when it comes to interaction, because it involves two big taboos in western societies: death and religion [13]. Those taboos can be clearly seen in U28's speech: "When accessing this memorial, although it was a fictional one, I felt uncomfortable, as these are very gloomy things".

As to the candles that enhance the reputation of a memorial in iHeaven, U12 said: "I realized different candles had different scores, and I wanted to give the highest scored one". U13 felt the same: "I felt I was contributing to enhance the score of that memorial". On the other hand, U08 criticized this functionality: "I don't think it is right to get points from homage, like a candle. Such a social network should not reward users (...)". This user also said it was disrespectful or incoherent to rate 
memorials or get score from this kind of interaction, which suggests gamification [1] is not well accepted by all usersin this kind of platform. Once again, death-related taboos influence the way users interacted with this tool, by preventing the association between bereavement and fun through gamification elements.

Creating Memorials. $86,20 \%$ of the users fulfilled the task of creating a memorial during the interaction test. Some of those who chose not to do so declared to feel uncomfortable or to be in a hurry. However, the user who said he was on a hurry stayed in the room of the test for many minutes after finishing the task-scenario, which might suggest he was actually uncomfortable or uninterested to create the memorial.

Most users created memorials dedicated to public people or fictional characters. U15 affirmed he "didn't feel he had the right to create a memorial for his dead cousins, even because he wouldn't have the time to administer those profiles and they would remain abandoned on the web". Again, the taboos towards death prevented people from interacting with this functionality of the software. U05 said: "There are people who accept death more easily than others, but for me (and I believe for many people), it is upsetting to think about that. No one thinks of his own grave". U24, when talking about this issue, shows the death-related taboo which founds culture, by separating what is profane and what is sacred [13]: "I felt as if I were doing digitally something that should only be done in the real world".

Many of the users who created memorials for friends said they have a mixed feeling of missing and sadness, for remembering the deceased person. U25 affirmed: "Creating a memorial made me feel very said, as I missed that person". On the other hand, U28 felt consoled when he created a memorial: "I felt as if I were sharing my sorrow for losing someone important, and as if I were giving to others the opportunity to express their feelings too".

Two users created memorials for relatives. U29, who created a memorial for his grandfather, stated: "Creating a memorial was pleasant, as I remembered a dear person. I thought of pleasing other relatives of mine with that deed, as a digital memorial overcomes all distances. However, picking photos was pretty sad". To select those pictures, U29 searched profiles on other social networks and showed on Youtube a song composed by his deceased grandfather.

It is important to say that all those actions were done by free will and were probably triggered by strong emotions raised by the interaction with this application.

Comparison between Virtual and Physical Memorials. The last part of the questionnaire addressed the comparison between virtual and physical memorials. Users were asked about the symbolic value of a virtual memorial if compared to a physical one. $65,51 \%$ of the users said both memorials have the same value, whereas $31,03 \%$ found the digital ones less symbolic and 3,44\% considered the digital ones as more symbolic. About this, U29 said: "In my opinion, a physical memorial has more sentimental value, but there is no conflict with the digital one, which is a complement in fact". 
When asked about the comparison between real-world and digital-world homage, $37,93 \%$ of the users said they have the same value. On the other hand, $48,27 \%$ stated the virtual ones are less meaningful, whereas $13,79 \%$ said they have more meaning. Most of those who considered virtual homage as more meaningful explained that this kind of homage rates the image of the deceased, as stated by U02: "It is as if I were telling everyone that person influences/influenced my life". Likewise, U04 said: "It could show other people my feelings for the dead". By their speeches, such users emphasize the wish for eternizing the reputation of the deceased.

The last question posed to users was if they wished for a physical memorial, a digital one, or both, when they died. $27,58 \%$ said they did not care about it; $31,03 \%$ said they wanted either a physical or a digital one; $10,34 \%$ did not want any memorial; 27,58\% preferred a digital one; and only 3,44\% wished for a physical one. Besides the greater range of people who may access a digital memorial, U06 presented another reason why so many users preferred the digital one: "as for digital memorials, there are no time or distance barriers for paying homage to a dear deceased person".

\section{Conclusion}

Memorials are traditionally physical constructions or material tributes to the honor of the dead, especially famous people or those who played an important historical role in a society. However, digital memorials provide us with a new device for mourning, which entails different cultural practices, including digital tributes, digital mourning and even digital prayers. They are also more democratic than physical memorials, once any user with access to the internet can create a memorial for a friend, a relative, or a public person. Actually digital memorials can even be created to celebrate the death of someone who did not really exist, like a fictional character, or the fictional death of someone who is still alive in the real world.

Digital memorials also permit that people transcend time and distance limits when it comes to mourning, which warrants the possibility of eternizing someone's memory on an online platform. Most respondents who participated in this research considered this democratic aspect of virtual memorials as something good.

Considering the digital memorials recently launched in Brazil, the respondents said they have some expectations concerning the use of those platforms as social networks. For example, many users said they would be interested in rating the deceased person's reputation, thus showing to others how important that person was. Likewise, if in the past memorials were important for religious and spiritual reasons, nowadays digital memorials allow for the online exposure of the dead persons' legacy, such as photos and videos, which poses privacy challenges to these platforms.

Another challenge we can infer from the data herein analyzed consists in finding an ethical way to approach death and the deep feelings it triggers in the users. For example, after visiting the memorial of a boy, U15 said: "I felt very sad when I saw his mother still posting many messages twenty years after his death". Modeling this kind of application demands a reflection on how far users, designers and researchers can go when dealing with such complex issues. 
From the verbal expressions throughout the interaction test and from the answers to the questionnaire, we could gather verbal data on how users felt when interacting with those applications. Many of them called that interaction "weird", or "bizarre", vague expressions for something that may be hard to name. Death-related taboos may hinder users' reflection on those applications or even prevent them from using some of the functionalities. This is what led such a significant amount of users not to create a memorial profile or click the button "pray" in the platform.

Designing virtual applications for users' interaction about death is also challenged by the need to model processes in which most users feel comfortable and satisfied, irrespective of their religion and death-related taboos. Therefore, the cultural diversity inherent to online mourning must be respected. Besides, it is also important to consider how to protect the deceased from libel and how to identify if the virtual death corresponds to a physical one.

The applications herein analyzed model the concept of digital memorials and tributes to the deceased, but they do not provide the user with the volitional element [12]. As it was suggested by U24, "it would be interesting if the user could choose whether or not he wanted that his profile on the social network turned into a memorial profile after his death". So far, if a user of those platforms dies, both his profile and the memorials he administers will be out of administration.

One of the difficulties found in this research is that the users were not bereaved; they were only faced with a platform to pay homage to unknown or public people. Therefore, new researches are necessary with really bereaved users. Besides, the platform iHeaven is a beta version, which caused some bugs and slowness and sometimes hindered interaction.

Future researches must be done with users from different generations, so as to identify whether they have different feelings towards this kind of interaction. Furthermore, it is important to bring other fields to dialogue in this research, such as Theology, Philosophy and Law. These issues demand deeper and more interdisciplinary studies, also employing different methodologies. For example, as many users found it hard to verbally express how they felt when interacting with those memorials, due to the deep feelings they evoke, other techniques must be used to collect data from body and facial language.

\section{References}

1. Alves, F.P., Santana, E.C., Maciel, C., Anacleto, J.: A rede social móvel Foursquare: uma análise dos elementos de gamificação sob a ótica dos usuários. In: Workshop Proc. WAIHCWS 2012 (2012) (in Portuguese), http://ceur-ws.org/Vol$980 /$ paper 3. pdf

2. American Heritage Dictionary of the English Language, http://education. yahoo.com/reference/dictionary/entry/memorial

3. Barbosa, S.D.J., da Silva, B.S.: Interação Humano-Computador. Elsevier, Rio de Janeiro (2010) (in Portuguese)

4. Braman, J., Dudley, A., Vincenti, G.: Death, Social Networks and Virtual Worlds: A Look Into the Digital Afterlife. In: Proc. SERA 2011, pp. 186-192. IEEE Computer Society, Washington, DC (2011) 
5. Brasil: Conselho Nacional de Saúde. Resolução 466/2012. Diretrizes e Normas Regulamentadoras de Pesquisas Envolvendo Seres Humanos (in Portuguese), http: / / conselho.saude.gov.br/resolucoes/2012/Reso466.pdf

6. Becker, S.H., Knudson, R.M.: Visions of the dead: Imagination and mourning. Death Studies 27, 691-716 (2003)

7. Hallam, E., Hockey, J.: Death, Memory, and Material Culture, Oxford, New York (2001)

8. IBGE: Censo Demográfico (2010) (in Portuguese), ftp://ftp.ibge.gov.br/ Censos/Censo_Demografico_2010/Caracteristicas_Gerais_Religia o_Deficiencia/tab1_4.pdf

9. Iheaven (in Portuguese), http: / / www . iheaven. me

10. Kern, R., Abbe, E.F., Gil-Egui, G.: R.I.P.: Remain in perpetuity. Facebook memorial pages. Telemat. Inf. 30(1), 2-10 (2013)

11. Lopes, A., Maciel, C.: Memoriais Digitais: Morte e manifestação do luto nos ambientes virtuais. In: Anais ERI-MT 2010 (2012) (in Portuguese)

12. Maciel, C.: Issues of the Social Web interaction project faced with afterlife digital legacy. In: Proc. IHC+CLIHC 2011, pp. 3-12. ACM Press (2011)

13. Maciel, C., Pereira: The Influence of Beliefs and Death Taboos in Modeling the Fate of Digital Legacy Under the Software Developers'View. In: CHI 2012 Workshop Memento Mori (2012), https://sites.google.com/site/chi2012eol/acceptedpapers / chi2012EAMacielandPereira.pdf?attredirects $=0$

14. Maciel, C., Roque, L., Garcia, A.C.B.: Interaction and communication resources in collaborative e-democratic environments: The democratic citizenship community. Information Polity 15, 73-88 (2010)

15. Massimi, M., Baecker, R.M.: Dealing with death in design: developing systems for the bereaved. In: Proc. of the CHI 2011, pp. 1001-1010. ACM Press, New York (2011)

16. Massimi, M., Odom, W., Banks, R., Kirk, D.: Matters of life and death: locating the end of life in lifespan-oriented HCI research. In: Proc. of CHI 2011, Vancouver, BD, pp. 987996. ACM Press (May 2011)

17. Massimi, M., Baecker, R.R.: A death in the family: opportunities for designing technologies for the bereaved. In: Proc. CHI 2010, pp. 1821-1830 (2010)

18. Memorial Digital (in Portuguese), http://www. memorialdigital.com.br

19. Mims, C.: When we Die: The Science, Culture, and Rituals of Death. St. Martin's Press, New York (1998)

20. Nielsen, J.: Why You Only Need to Test with 5 Users, http: / www. nngroup.com/ articles/why-you-only-need-to-test-with-5-users/

21. Nielsen, J., Landauer, T.K.: A mathematical model of the finding of usability problems. In: Proc. INTERCHI 1993, pp. 206-213 (1993)

22. Pressman, S.R., Lowe, D.: Engenharia Web, 1st edn. Livros Técnicos e Científicos (2009) (in Portuguese)

23. Ricoeur, P.: Memory, History, Forgetting. University of Chicago Press (2004)

24. Riechers, A.: The Persistence of Memory Online: Digital Memorials, Fantasy, and Grief as Entertainment. In: Maciel, C., Pereira, V.C. (eds.) Digital Legacy and Interaction: PostMortem Issues. HCI, pp. 49-61. Springer, Switzerland (2013)

25. Saudade Eterna (in Portuguese), http : / /www. saudadeeterna. com. br

26. Smith, G.: Social software building blocks (2007), http://nform.ca/ publications/social-softwarebuilding-Block

27. Tapscott, D.: A hora da geração digital: como os jovens que cresceram usando a internet estão mudando tudo, das empresas aos governos. AgirNegócios, 448 (2010) (in Portuguese) 